\title{
Estrategias de fortalecimiento de capacidades para adaptación al cambio climático en comunidades de alta montaña de la subcuenca del río Quillcay en Áncash, Perú
}

\author{
Strategies for capacity building for adaptation to climate change in high \\ mountain communities of the Quillcay sub-basin in Ancash, Peru
}

Jenny Menacho Agama ${ }^{1}$

\section{RESUMEN}

El estudio tiene por objetivo conocer el rol que desempeña el fortalecimiento de capacidades para adaptación al cambio climático en ecosistemas de alta montaña como la subcuenca del río Quillcay. Propone estrategias viables que busquen resultados más eficaces. Para indagar sobre los temas priorizados y las mejores estrategias para fortalecer capacidades en adaptación de interés para los actores sociales de la subcuenca, se realizó un estudio exploratorio descriptivo por ser este tema poco estudiado en el contexto de ecosistemas de alta montaña y ámbitos de glaciares como las de la subcuenca Quillcay. La metodología adaptativa y flexible ha permitido constatar que el tema de cambio climático relativamente nuevo se reconoce el rol de fortalecimiento de capacidades prioritario en la percepción de los actores y útil especialmente para los campesinos, quienes sienten de manera directa los efectos del cambio climático. No existe una estrategia única, mas sí sugerencias de algunas pautas metodológicas para llevar adelante procesos de fortalecimiento de capacidades más efectivas.

Palabras clave: fortalecimiento de capacidades; adaptación al cambio climático; ecosistemas de montaña.

\footnotetext{
1 Fondo Verde Internacional, Perú. CEPES - Universidad de Michoacán, México.

(C) Los autores. Este artículo es publicado por la Revista Aporte Santiaguino de la Universidad Nacional Santiago Antúnez de Mayolo. Este es un artículo de acceso abierto, distribuido bajo los términos de la Licencia Creative Commons Atribución-NoComercial-CompartirIgual 4.0 Internacional. (http://creativecommons.org/licenses/ by-nc-sa/4.0/), que permite el uso no comercial, distribución y reproducción en cualquier medio, siempre que la obra original sea debidamente citada.
} 


\begin{abstract}
The study aims to understand the role played by the strengthening of capacities for adaptation to climate change in high mountain ecosystems such as the sub-basin of the Quillcay River, proposing viable strategies that seek more effective results. To investigate the prioritized topics and the best strategies to strengthen capacities in adaptation of interest for the social actors of the sub-basin, an exploratory descriptive study has been carried out since this subject is little studied in the context of high mountain ecosystems and areas of glaciers such as those of the Quillcay sub-basin. The adaptive and flexible methodology has made it possible to confirm that the relatively new issue of climate change recognizes the role of priority capacity building in stakeholder perception and is especially useful for farmers who directly feel the effects of climate change. There is no single strategy, but rather suggestions of some methodologies guidelines to carry out more effective capacity building processes.
\end{abstract}

Keywords: capacity development; climate change adaptation; mountain ecosystems.

\title{
INTRODUCCIÓN
}

La investigación se enmarca en el tema de adaptación al cambio climático (ACC), que según los entendidos se hace cada vez más presente en la agenda de los investigadores, políticos y encargados de programas conscientes de que el cambio climático es real y amenaza con socavar la sostenibilidad social y ecológica del planeta.

De manera genérica la capacidad de adaptación, según el PNUD (2009: 8) es referida como «la capacidad de un sistema para ajustarse a los cambios, aprovechar las oportunidades y tolerar las consecuencias», y en particular la capacidad de adaptación según, el Grupo Intergubernamental de Expertos sobre el Cambio Climático - IPCC (2001), implica que la capacidad de cualquier sistema puede ajustarse a los cambios de la variabilidad climática y los cambios extremos para lidiar con los daños potenciales, soportando las consecuencias negativas o aprovechando las consecuencias positivas.

Estudios sobre cambio climático consideran que existe una gran demanda por información sobre cómo enfrentar la adaptación, y según Prettengell (2010: 45) las medidas de adaptación «no son necesariamente nuevas pero sí lo son el camino seguido para identificarlas y los cambios transformacionales que pretenden logran». En ese sentido lo que cuenta son las experiencias y el conocimiento adquirido en la práctica y son pocas las experiencias explicitadas, diversificadas en materia de tecnologías blandas o duras, con posibilidad de replicarse para zonas similares.

En ecosistemas de alta montaña y particularmente en la cuenca del Santa de la región Áncash, se han desarrollado diversas iniciativas desde organizaciones de cooperación (ONG nacionales e internacionales) en coordinación con organismos del Estado, con 
mayor protagonismo los años 2011 a 2014, pero con escasez de investigaciones y evaluaciones publicadas sobre el componente específico de capacidades, desconociéndose aun hasta qué punto se logran fortalecer o construir capacidades y conocimientos que satisfacen las demandas locales, por ejemplo, en la gestión del riesgo de desastres y ACC de manera efectiva.

Han actuado en la zona diversos proyectos e intervenciones específicas y sectorizadas de corto y mediano plazo, pero al parecer no logran instaurarse bien ni sentar las bases para impactar en la capacidad de los sujetos o poblaciones objetivo, en adición, con el telón de fondo caracterizado por la ingobernabilidad en un contexto político de inestabilidad de autoridades en el Gobierno Regional de Áncash que aún constituye un pasivo, a causa de la corrupción de funcionarios con perjuicio económico por pagos para obras inconclusas o incrementos sin justificación.

En este contexto el estudio propuesto, tiene por objetivo general, conocer el rol que desempeña el fortalecimiento de capacidades para adaptación al cambio climático en ecosistemas de alta montaña como la subcuenca Quillcay, proponiendo estrategias viables que busquen resultados más eficaces pudiendo ser útiles para otros contextos.

\section{MATERIALES Y MÉTODOS}

\section{Zona de Estudio}

La subcuenca del río Quillcay con un área de $250 \mathrm{Km}^{2}$ aproximadamente, se localiza en los Andes Centrales del Perú, con presencia de glaciares tropicales y es una de las principales aportantes de la cuenca del río Santa. Limita por el norte con la subcuenca de Llaca y por el sur con la subcuenca de Pariac. La zona presenta dos estaciones bien marcadas durante el año, una lluviosa y otra seca (Sotelo et al. 2016: 57)

La subcuenca Quillcay es muy singular históricamente, debido a la recurrencia de fenómenos catastróficos (como avalanchas y aluviones) y la presencia en su delta aluvial de la ciudad de Huaraz, capital del departamento de Áncash. En la actualidad, a raíz de la Ley N $^{\circ} 30612$ que declara de preferente interés nacional y necesidad pública la protección, conservación y prevención de riesgos de la laguna Palcacocha, se torna mayor el interés por la subcuenca de Quillcay. En ese sentido es un foco de interés para autoridades e investigadores, por ser una de las subcuencas más sensibles a los efectos del cambio climático, aumentando su vulnerabilidad por el retroceso glaciar y la pérdida de estabilidad de las masas de hielo, por lo que investigadores como Mallqui (2014), sugieren la inminente disminución de la oferta hídrica de la subcuenca en los próximos años. 
Con una diversidad de actores por la intervención con diferentes actividades y con énfasis en la gestión del riesgo de desastres, es un área de estudio y monitoreo constante por entidades e instituciones privado-públicas de gobierno regional y organismos adscritos de gobierno central entre otros.

\section{Diseño de la investigación}

La investigación es de tipo exploratoria descriptiva, a partir de la información que se recoge en el proceso para el cumplimiento de los objetivos, cuya operacionalización ha permitido realizar una descripción superior de los hallazgos acerca de la información recolectada, por ser el fortalecimiento de capacidades en ACC un tema poco explicitado y explorado en la zona de estudio. Asimismo, pone énfasis en tres experiencias que más se han desarrollado como punto de partida, para buscar recomendaciones que alimenten propuestas de mejora de fortalecimiento de capacidades para ACC en contextos de ecosistemas de alta montaña y ámbitos de glaciares como las de la subcuenca Quillcay pudiendo ser útiles para actores en otros contextos.

En ese sentido la perspectiva de la investigación es describir cómo el tema del cambio climático ha sido trabajado por las instituciones, y de manera específica bajo que modelos, mecanismos o estrategias se ha realizado el fortalecimiento de capacidades para ACC, con el fin de identificar expectativas, recomendaciones y desafíos desde perspectivas diferentes de los grupos de interés en el tema.

Autores como Hernández et al. (2010: 80) mencionan que los estudios de carácter descriptivo «buscan especificar las propiedades, las características y los perfiles de personas, grupos y fenómenos; es decir únicamente pretenden medir y recoger información de manera independiente o conjunta sobre los conceptos o variables, sin indicar cómo se relacionan entre ellas». Por lo tanto, el enfoque empleado en la investigación planteada es de carácter cualitativo. El enfoque cualitativo «utiliza la recolección de datos sin medición numérica para descubrir o afinar preguntas de investigación en el proceso de interpretación» (Hernández et al., 2010: 7). En ese sentido, las características de la población investigada ${ }^{2}$ la constituyeron diversos actores implicados o con conocimiento sobre las iniciativas de fortalecimiento de capacidades para ACC, los que según su grado de importancia se han clasificado en condición de implicancia directa o indirecta, tal como se muestran en la siguiente tabla.

\footnotetext{
2 El trabajo de campo de la investigación se apoyó en el "servicio de consultoría para la formulación e implementación de un programa de gestión del conocimiento y fortalecimiento de capacidades orientado a fomentar la investigación científica y su aplicación" a cargo de la autora ejecutada por el INAIGEM, de octubre a diciembre 2017, oportunidad para tomar contacto con la población y los actores de la parte alta de microcuencas en la zona de estudio.
} 
Tabla 1. Actores clave para entrevistas en la subcuenca de Quillcay

Directo Indirecto

Grado de

Instru-

ACTORES importan-

mento/

cia

Cantidad

(De 1-4)

(E-O-

$\mathrm{R}-)^{*}$

1. Gobierno Regional de Ancash: GRN / DRE - Gerentes; Jefe INDECI.

E: 3

2. Municipalidades de nivel provincial y distrital -Alcalde, Gerente Gestión del

E: 3

Riesgo y Especialista GdR)

3. Ministerio del Ambiente: INAIGEM

(4) - Dirección General de Cambio Climático y Dirección de Participación

4. SERNANP-Jefe PNH - Especialista comunicaciones

$\mathrm{x}$

1

E: 2

5. CARE - Central Lima / Coordinación Nacional y Zonal Huaraz Proyecto Glaciares +

6. TMI - Central Lima / Coordinación Huaraz -Coordinadores y Especialistas

7. Mancomunidad Waraq (Distritos Huaraz e Independencia). Presidente

8. Dirigentes y líderes de Centros Poblados en Comunidades campesinas de la Subcuenca Quillcay

9. Universidad Nacional Santiago Antúnez de Mayolo - Huaraz. Docentes especialistas $\mathrm{x}$

X

$\mathrm{x}$
1

$\mathrm{E}: 1$

E:10

1

O: 4

R:3

E:2

*E: Entrevista individual, O: Observación; R: Reuniones de indagación y análisis (Taller de aprendizaje) 
El tipo de muestreo fue al azar y por conveniencia, seleccionándose como referencia tres iniciativas en las cuales se han atribuido mayor experiencia en el tema, y convocaron a diversos actores, los cuales constituyeron integrantes de un primer grupo de estudio. Estos son los proyectos: Construyendo capacidades cientificas, sociales e institucionales para el manejo y reducción del riesgo de avalanchas de lagos glaciares en la Cordillera Blanca, Perú (Zinmer et al. 2017); Implementación de medidas de adaptación al cambio climático en la subcuenca del río Quillcay (IMACC-MINAM/ BID), en adelante proyecto IMACC en Quillcay, y el Proyecto Glaciares + - CARE/COSUDE.

Para el segundo grupo de estudio, se consideró que según el método bola de nieve desde las experiencias seleccionadas podrían identificar y referir a actores con mayor experiencia y conocimiento en el tema, mas dado el cambio de autoridades de los sectores público y privado, muchos de los potenciales entrevistados ya no residían en la zona o era difícil contactarlos, por lo que se decidió incluir a otros actores de entidades que consideraban incorporar dentro de sus estrategias el fortalecimiento de capacidades para ACC y poseían una inicial experiencia en el tema como el Instituto Nacional de Investigación en Glaciares y Ecosistemas de Montaña - INAIGEM. El tercer grupo estará conformado por población usuaria del servicio de capacitación o fortalecimiento de capacidades en ACC, tales como líderes, directivos y/o autoridades, mujeres y varones de centros poblados o comunidades.

Como resultado de este proceso se planificó la realización de 33 entrevistas individuales, 5 experiencias para observación participativa y 3 reuniones de aprendizaje. No obstante para el desarrollo de la investigación se realizará preliminarmente el análisis en base a 20 entrevistas pertenecientes a autoridades, funcionarios y especialistas de entidades públicas y ONG del primer y segundo grupo.

\section{RESULTADOS Y DISCUSIÓN}

El resultado del análisis reconoce que el tema de cambio climático es relativamente nuevo en la agenda de los actores públicos, ya que son las ONG como el Instituto de Montaña y CARE Perú, quienes empezaron su abordaje en Huaraz. Un especialista del INAIGEM con larga trayectoria ejecutando proyectos de desarrollo, manifiesta que: «Antes en 2007 a 2009, éramos poquitos de manera independiente andábamos hablando de adaptación al cambio climático, ahora a diez años, se ha vuelto prioritario para muchas instituciones... () Por los años 2010 con la segunda comunicación, en ese tiempo quien más involucrado era fue el Instituto de Montaña y posiblemente ahora el SERNANP, aunque se encuentra más arriba en la cuenca, más alejado de los usuarios de riego» (Helder Mallqui, 45, Huaraz, 26/01/2018-HM). 
En esa misma línea, otro especialista del IM refiere que: «El tema de gestión de proyectos asociados a cambio climático los ha visto en Carhuaz CARE, pero en la subcuenca no lo ha hecho con comunidades, sino financiar consultorías de otros para asesoría a las autoridades en la parte baja, como por ejemplo para la Estrategia Regional de Cambio Climático» (Eduardo Castro, Marcará, 09/02/2018-EC).

Un excoordinador del proyecto IMACC manifiesta que: «Desde el 2012 al 2014 el proyecto IMACC trabajó en la zona como un impulso a la Estrategia Regional de Cambio Climático (ERCC), cuando era Gerente el Ing. Erick Mautino Minaya. En este proyecto de manera explícita se trabajó con medidas de adaptación al cambio climático» (Ricardo Villanueva, 42, 25/01/2018-RV).

Hay distinciones entre lo que implica hacer mitigación y adaptación al cambio climático (ACC) por parte de los entrevistados, siendo contundentes las afirmaciones de que la mitigación se relaciona directamente con la reducción de GEI y les corresponde trabajar a los países más industrializados, mientras que la adaptación implica un trabajo de respuesta desde países en desarrollo como el Perú y se relacionan con nuevas prácticas en un entorno cambiante.

$\mathrm{Al}$ respecto un gerente del gobierno regional opina que: «Mitigación implica ejecutar proyectos de forestación para captura de CO2, tiene que ver con GEI, mientras que adaptación tiene que ver con las personas que se tienen que ir adaptando al entorno» (Sandro Castillo, Huaraz, 22/11/2017-SC).

Las manifestaciones del cambio climático y sus efectos, lo vienen sintiendo los campesinos, y un testimonio al respecto considera que «los campesinos en Santa Cruz manifestaron que ahora el maíz crece en partes más altas y urge además atender el problema del agua con soluciones de gestión integrada, un aspecto complejo porque en el marco de cambio climático está trayendo más conflictos, la gestión del agua es pre requisito para la paz social» (CP). De modo similar otro especialista del IM considera que «Trabajar adaptación es lo principal en la zona, son los campesinos que están sufriendo ya las consecuencias de retroceso glaciar y sufren el cambio de clima, hay algo intrínseco, por eso promover iniciativas locales es importante y urgente» (EM).

Para el representante de gobierno regional: «Fortalecer, es cuando ya existe algo, desarrollar es cuando se tiene que comenzar. En esta zona se tiene que desarrollar, porque las personas, por ejemplo la falta de agua no lo ven como un problema relacionado a cambio climático» (SC). Los testimonios de los siguientes entrevistados dan cuenta de lo señalado: «Si vas a fortalecer capacidades es porque hay algunas. Si hay conocimiento previo tradicional debiera ser revalorado» (David Ocaña, 65, Huaraz, 20/09/2018 DO). «Fortalecer capacidad, implica primero que cada persona va a tener capacidades, se trata de rescate originario y darle más información para cumplir su misión. Se asume que tienen el conocimiento» (Helder Mallqui, 45, Huaraz, 26/01/2018-HM). 
Considerado como proceso que debiera ser reiterado y sostenible. «Fortalecimiento de capacidades es cuando se trabaja el riesgo con simulacros reiterativos, hacer de día y de noche (...) capacitaciones de manera permanente, secuencial y en aspectos específicos. Mientras que desarrollo implica trabajar donde antes no se pudo o hubo, es empezar de cero» (Ricardo Villanueva, 42, 25/01/2018-RV). «Fortalecer está orientado a hacer algo más sostenible en el tiempo» (Helder Mallqui, 45, Huaraz, 26/01/2018-HM).

Implica además, tener resultados o evidencia. Entrevistados con larga experiencia de campo opinan que: «Si ya existen capacidades, entonces se tiene que llevar algo más de lo que ellos ya saben, (...) porque no se va a fortalecer si el sujeto no ve beneficio adicional» (David Ocaña, 65, Huaraz, 20/09/2018-DO).

«Se tiene que hacer a través de un proyecto con uso de la práctica y algo muy a largo plazo, porque para mí no sirve solo talleres y charlas» (...) «Si ellos necesitan un riego tecnificado, para mí el camino ideal es ir a la comunidad, pasar tiempo y en detalle verificar con ellos las dificultades técnicas, todas las condiciones» (Anais Zimmer, Huaraz, 29/01/2018-AZ).

«No hay fortalecimiento de capacidades efectivo si este no va acompañado de un proyecto o acción que se concretice en algo útil para el campesino» (...). «El campesino quiere y debe ver algo concreto» (Cesar Portocarrero, Huaraz, 07/02/2018-CP).

Todos los entrevistados consideran la importancia del fortalecimiento de capacidades en las acciones de desarrollo en cualquiera de sus formas. Por ejemplo los del gobierno regional consideran que: «Es fundamental el FC, así se logra cambio en las personas. Si no se hace prevención de lo contrario no se espera una buena reacción» (Sandro Castillo, Huaraz, 22/11/2017 -SC). Entrevistados del INAIGEM opinan que: «Es prioritario, pero si tienes claro el grupo meta, dirigido a familias, comunidad y sectores o instituciones, si esto es claro vas acomodando tus paquetes de que vas a rescatar, que vas a adaptar» (DO).

Otros miembros del Instituto de Montaña consideran que es transversal porque: «Es a todo nivel de todos los actores, sobre todo a nivel de municipalidades y son los que tienen otras prioridades. Es una época en que la población busca beneficio inmediato, se ve siembra y cosecha de agua, ellos ven el día a día» (EC), además posibilita el empoderamiento porque: «Si la gente ha hecho todo el proceso, y esta capaz de replicarlo es que se siente empoderado, que va a difundir lo que ha aprendido y están fuertes, que han aprendido que si hay que vencer barreras las pueden pasar» (AZ).

\section{Acciones, actividades e instrumentos no necesariamente para ACC}

Los antecedentes de trabajo en la zona, hacen referencia a instituciones como el Parque Nacional Huascarán (PNH) para conservación de Áreas Naturales Protegidas con 
reforestación de especies nativas y conformación de Comités de Usuarios de Pastos, la construcción de infraestructura para captación y canalización de agua en Quillcayhuanca, una obra paralizada y ejecutada por la compañía minera ANTAMINA, con estudios para analizar la calidad del agua y una labor más permanente del Instituto de Montaña en la microcuenca de Shallap y centro poblado de Coyllur para el tema en aspectos de bioremediación del agua, recuperación de bofedales en convenio con la Universidad Nacional Agraria La Molina.

Las acciones que más se han hecho en la subcuenca Quillcay, no necesariamente con el propósito de fortalecer capacidades, ha sido en el proceso de gestionar el riesgo alrededor de la laguna Palcacocha (2012 al 2014). Estas van desde la intención de fortalecer capacidades institucionales con asistencia técnica a las autoridades regionales, distritales y comunales, hasta estudios y diagnósticos en convenio con la Universidad de Texas y TMI tales como, por ejemplo, «Estudios de Infraestructura de Riego y Modelamiento Hidrológico» y «Diagnóstico social de los Comités de Usuarios de Pastos de la Subcuenca Quillcay». Así mismo se menciona la «Consultoría para Servicios Eco sistémicos, implicando talleres y reuniones con diversas autoridades de la microcuenca Quillcayhuanca» (RV). En esta misma microcuenca o quebrada, la Municipalidad de Independencia desarrolló un proyecto de reforestación con pino (ejecutado desde el 2011 al 2014) y en convenio con el PNH, con especies nativas de quenuales (Polylepis spp.).

En el año 2016, el INAIGEM elabora el "Proyecto Integral de Seguridad de la Laguna Palcacocha" que en opinión de un entrevistado quedó en documento, no obstante la organización en la ciudad de Huaraz del "Congreso Mundial de Glaciares", marcó un hito por la difusión de retroceso y peligros glaciares de utilidad para INDECI y CENEPRED. Posteriormente, ingresa a un sector de la microcuenca Quillcayhuanca (Tayacoto) para realizar estudios en área reforestada con pino y monitoreo de la calidad de agua en las tres microcuencas de la subcuenca.

El estudio identificó dificultades de tipo normativo y de gestión de los gobiernos regional y local, por ejemplo los directivos del gobierno regional reconocen que «No es suficiente una ordenanza con compromisos, lo demuestra la Estrategia Regional frente al Cambio Climático que se quedó allí, por no tener presupuesto. No tiene un Programa Presupuestal para ACC» (SC).

Por otra parte, el cumplimiento de compromisos de corto plazo por las ONG o proyectos que no han resuelto problemas de fondo en la percepción de los entrevistados, aun cuando productos de dichas acciones se han explicitado en documentos e informes y algunas publicaciones realizadas por el Instituto de Montaña o CARE. En opinión del gobierno regional, «Las ONG generan dinamismo y afán de mostrar metas cumplidas y resultados, pero no respondemos porque del universo de trabajadores del 
gobierno regional solo un 8\% apoya» (SC). Considera además que «Se ha introducido adaptación al cambio climático medianamente, pero el problema son las personas porque se está perdiendo la voluntad de generar el verdadero cambio» (SC).

Se sostiene que: «Usualmente lo que más se hace es querer transferir conceptos de cambio climático a las comunidades y las tratan como si ellas no supieran nada (...). No es tan fácil, tienes que ligarlo a un proceso. Te vas a encontrar con campesinos que si le preguntas lo que se está haciendo, te gusta o no te gusta, no te van a decir o te va a decir esto ya lo sabía» (DO).

Al parecer, las expectativas no cubiertas imposibilitan la apropiación de "nuevos" conocimientos, en la opinión de miembros del INAIGEM cuando se sostiene que: «En cuanto a cambio climático y gestión del riesgo conocen los términos pero no los hacen suyos. No es parte de su tema, no le toman importancia porque sea como sea tienen agua, no es crítico en la Cordillera Blanca, a diferencia de quienes no tienen agua han aprendido a racionalizar (Cordillera Negra), es menos crítico el peligro en esta zona» (HM). Y en opinión de un consultor: «El problema es que los técnicos no hablan en lenguaje sencillo, muchos tecnicismos por ejemplo se habla de 'ablación' cuando se puede decir 'aflojamiento del hielo' es correcto y simple» (CP).

Como parte del análisis de las entrevistas, dos entrevistados mencionaron las carencias de competencias que incluyan habilidades y actitudes de los especialistas o promotores que van al campo cuando relatan lo siguiente: «cuesta encontrar los antecedentes, y no saber valorar la experticia de otros, no les gusta a los ingenieros hacer talleres, prefieren estar sentados en la PC o trabajando en gabinete procesando sus datos, no se sienten cómodos y desconfían de sus capacidades para hacerlo» (Helder Mallqui, 45, Huaraz, 26/01/2018-HM).

«Yo soy una técnica, pero aprendí a reconocer el trabajo de los demás que tienen una formación social para poder compartir y hacerme entender con la gente. Esto es algo que tienen las ONGs no sé si lo tiene el Estado» (Anais Zimmer, 30, Huaraz, 29/02/2018).

En esta lógica, el PNUD (2009: 48) considera la necesidad de "paquetes" articulados como medidas para fortalecer capacidades, servicios de desarrollo de capacidades a corto y mediano/largo plazo, los que van desde arreglos institucionales más eficaces, incentivos monetarios y no monetarios, capacitación a agentes en prestación local de servicios, hasta suministro de un diseño de "buenas prácticas" y otras, incluido el "fortalecimiento institucional o provisión de capacidades y sistemas locales para la comunicación con el público y mecanismos de retroinformación"; es decir, un conjunto de acciones interconectadas que logren sinergia para articular procesos de gestión hasta conseguir resultados. 
Las estrategias para fortalecer capacidades, aun cuando no han sido explicitadas o sistematizadas cobran sentido si se asume, según Torres (2008: 15), «la importancia de determinar el nivel de la capacidad de absorber los cambios (resiliencia) que tiene cada uno de los componentes de los ecosistemas implicados», y es notorio que el conjunto de componentes del caso estudiado, priman los aspectos físicos (manejo del agua, o estudio de suelos en una plantación de pinos) y biológicos (con la recuperación de bofedales o cultivo de microorganismos), pero poca referencia o visibilidad cobran los aspectos socioculturales y económicos.

\section{CONCLUSIONES}

El análisis muestra que, si bien se reconoce el rol fundamental que juega el fortalecimiento de capacidades para adaptación al cambio climático, el tema en general y particularmente con respecto a la adaptación es bastante reciente. A diez años de haberse creado el Ministerio del Ambiente en Perú, las acciones o intervenciones bajo este componente no terminan de consolidarse como una estrategia sistemática de largo alcance, siendo las ONG como CARE Perú y el Instituto de Montaña, que dada su experiencia pionera de trabajo en la zona de estudio, han iniciado el camino para experimentar o promover mecanismos de adaptación que al ser focalizadas, esporádicas o temporales no terminan de consolidarse y las iniciativas públicas con apoyo de cooperación y organismos multilaterales como el BID, aún no pueden servir de modelo o ser valoradas en toda su magnitud por los usuarios a quienes se dirige.

Los procesos y resultados factibles de intervenciones para ACC, pasan por obtener la claridad y delimitación en su accionar y voluntad en las instituciones para dar prioridad en sus programas de gobierno local y regional de manera más estructurada, de mediano y largo plazo incidiendo en el componente de fortalecimiento de capacidades a nivel de toda la subcuenca, el cual es considerado una prioridad adquiriendo un rol preponderante, especialmente las zonas altas de microcuencas con ecosistemas de cercanía a glaciares y lagunas de origen glaciar.

Existe necesidad de explicitar las mejores estrategias, diseñarlas de manera sistemática y probarlas bajo pautas metodológicas que tienen que ver con la especificidad de los temas con aspectos prácticos que sean de interés para la población objetivo, combinar los conocimientos técnicos y científicos con los tradicionales, construyendo lazos de confianza para fortalecer así, las capacidades locales de adaptación con resultados más eficaces. 


\section{REFERENCIAS BIBLIOGRÁFICAS}

Hernández, Roberto; Fernández, Carlos y Baptista, Maria del Pilar. 2010. Metodología de la investigación. Quinta edición. Ed. McGRAW-HILL / INTERAMERICANA EDITORES, S.A. DE C.V. México, DF.

IPCC. (2001). Climate Change 2001: Mitigation. Contribution of Working Group III to the Third Assessment Report of the Intergovernmental Panel on Climate Change. [Metz, B., O.R. Davidson, R. Swart, y J. Pan (Eds.)]. Cambridge University Press, Cambridge, Reino Unido y Nueva York, NY, Estados Unidos.

Mallqui, Helder E. 2014. Informe final. Evaluación de la infraestructura bidráulica y social en la subcuenca del rio Quillcay, Huaraz, Ancash. BID- MINAM PET 1168 (documento de uso interno).

PNUD. 2009. Desarrollo de capacidades: Texto básico del PNUD. <http://www.unpcdc. org/media/220984/capacity_development_a_undp_primer_spanish.pd.> [Consulta: 26-03-2016].

Prettengell, Catherine. 2010. Adaptación al cambio climático, Informe de investigación de Oxfam Internacional, abril 2010.

Sotelo, Ivonne y otros. 2016. "Caracterización climática de la precipitación en la subcuenca del río Quillcay”. Boletín Inaigem. Año 1, No.1. Huaraz: Instituto Nacional de Investigación en Glaciares y Ecosistemas de Montaña. 57-68.

Torres, Juan. 2008. Gestión del agua para enfrentar el cambio climático / Editores: César Portocarrero, Juan Torres, Anelí Gómez. Responsables de la sistematización del proyecto: Marlene Rosario, Pedro Ferradas, Alcides Vilela. Revisión Carlos de la Torre. Lima: Soluciones Prácticas-ITDG.

Zimmer, Anais y Montes, Daniel. 2017. Territorio Seguro y Resiliente. Características y oportunidades del territorio en un contexto de Cambio Climático Mancomunidad Municipal Waraq. Diagnóstico territorial, tendencia del clima, y Estrategias de Adaptación al Cambio Climático 2017. The Mountain Institute-TMI.

Recepción: 30/04/2018

Aceptación: 20/06/2018

\section{Correspondencia}

Jenny Luz Menacho Agama

jennymenacho@gmail.com 